\title{
Guidelines for Technical Regulations and Standardization of Turbine and Hydromechanical Equipment During Production and Exploitation
}

\author{
Miodrag Arsić ${ }^{\text {, Brane Vistać }}{ }^{1}$, Srđan Bošnjak ${ }^{2}$, Goran Bošković ${ }^{3}$, Zoran Savić ${ }^{1}$ \\ ${ }^{1}$ Institute for materials testing, Bulevar vojvode Mišića 43 Belgrade, miodrag.arsic@institutims.rs \\ ${ }^{2}$ Faculty of Mechanical Engineering, Kraljice Marije 16, Belgrade, sbosnjak@mas.bg.ac.rs \\ ${ }^{3}$ Faculty of Mechanical and Civil Engineering, Dositejeva 19, Kraljevo, boskovic.g@mfkv.kg.ac.rs
}

Turbine and hydromechanical equipment at hydro power plant "Đerdap 1" is subjected to service conditions which lead to degradation of materials and structures. Taking into account the frequency of failure occurrence and experience accumulated through long term testing and determination of state, it can be concluded that basic causes of equipment degradation are: oscillations, fatigue, corrosion, erosion and cavitation.

It is the responsibility of manufacturers of turbine and hydromechanical equipment to make sure that all vital parts and supporting structures are made in compliance with technical requirements, suitable standards and european directives that refer to structural integrity. Applicability of Pressure Equipment Directive 97/23/EC and Machinery Directive 98/37/EEC is especially relevant, due to the fact that certain parts and structures of turbine regulation system, lubrication system, turbine runner and measurement system are subject to one of the above mentioned directives.

This paper contains the overview and basic parameters of new and general approach to standardization and technical harmonization of hydro power plant equipment that refers to structural integrity.

Keywords: hydro power plant equipment, technical regulations, standardization, European Directives, structural integrity

\section{INTRODUCTION}

Strains at hydropower plant turbine and hydromechanical equipment originate during the production of components and equipment assembly (residual stresses), during the process of performing functional requirements in exploitation (stationary and dynamic loads) and during the disturbed process of exploitation (non-stationary dynamic loads). Taking into account unpredictable influence of corrosion, erosion and cavitation during exploitation, it's clear that strains at components and equipment as a whole can`t be expressed by a simple mathematical function.

Design, construction and putting into operation of the hydropower plant comprises complex tasks. A large number of details have to be defined, designed, carefully coordinated and realized in order for safety and costeffectiveness of the project to be ensured. If only a few of a large number of details get overlooked, underestimated or incorrectly perceived, significant complications can arise in a short period of time.

Pressure Equipment Directive is relevant for the manufacturers of equipment such as pressure vessels, tanks, heat exchangers, steam generators, boilers, industrial pipeline installations, safety equipment and pressure fittings.

Machinery Directive is relevant for all machines or mechanical devices such as: operating machines, all kinds of stationary or movable machines for processing and recycling, hand-guided or hand-held devices, pumps, compressors, testing machines, packing machines etc.
Also, this Directive refers to all movable mining machines and lifting machines.

In figure 1 part of hydro power plant "Djerdap 1" turbine equipment is presented, which is subject to a large number of Directives.

\section{CAUSES OF DAMAGE OCCURRENCE AND MATERIAL DEGRADATION DURING OPERATION}

Hydro power plants are interested in developing means of protection of turbine and hydromechanical equipment from undesirable occurrences, such as degradation of parent material and/or weld metal of parts and structures, caused by stresses and unpredictable influence of oscillations (vibrations), fatigue, corrosion, erosion and cavitation.

\subsection{Damaging and Degradation of Material Due to Variable Loads and Oscillations}

Excitation forces in hydro generator units originate from hydraulic, mechanical and electrical sources. Their values, obtained through testing, are being compared to standard and allowable values. Some countries and professional organizations suggest Standards based on material testing. If oscillation magnitudes meet the requirements of these Standards, normal operation and safety of the facility is ensured.

For the time being international Standards regarding the magnitude of oscillations do not exist. Although Standards which regard rotating mechanisms include oscillation magnitudes for various machine types, 
consideration of oscillation magnitudes for hydraulic turbines has to take into account the following characteristics:

- low speed operation (general Standard is applicable only for high velocities),
- vertical shaft configuration (Standards are based on horizontal shafts),

- low speed turbines and large diameter turbines, - unequal or pulsating oscillations.

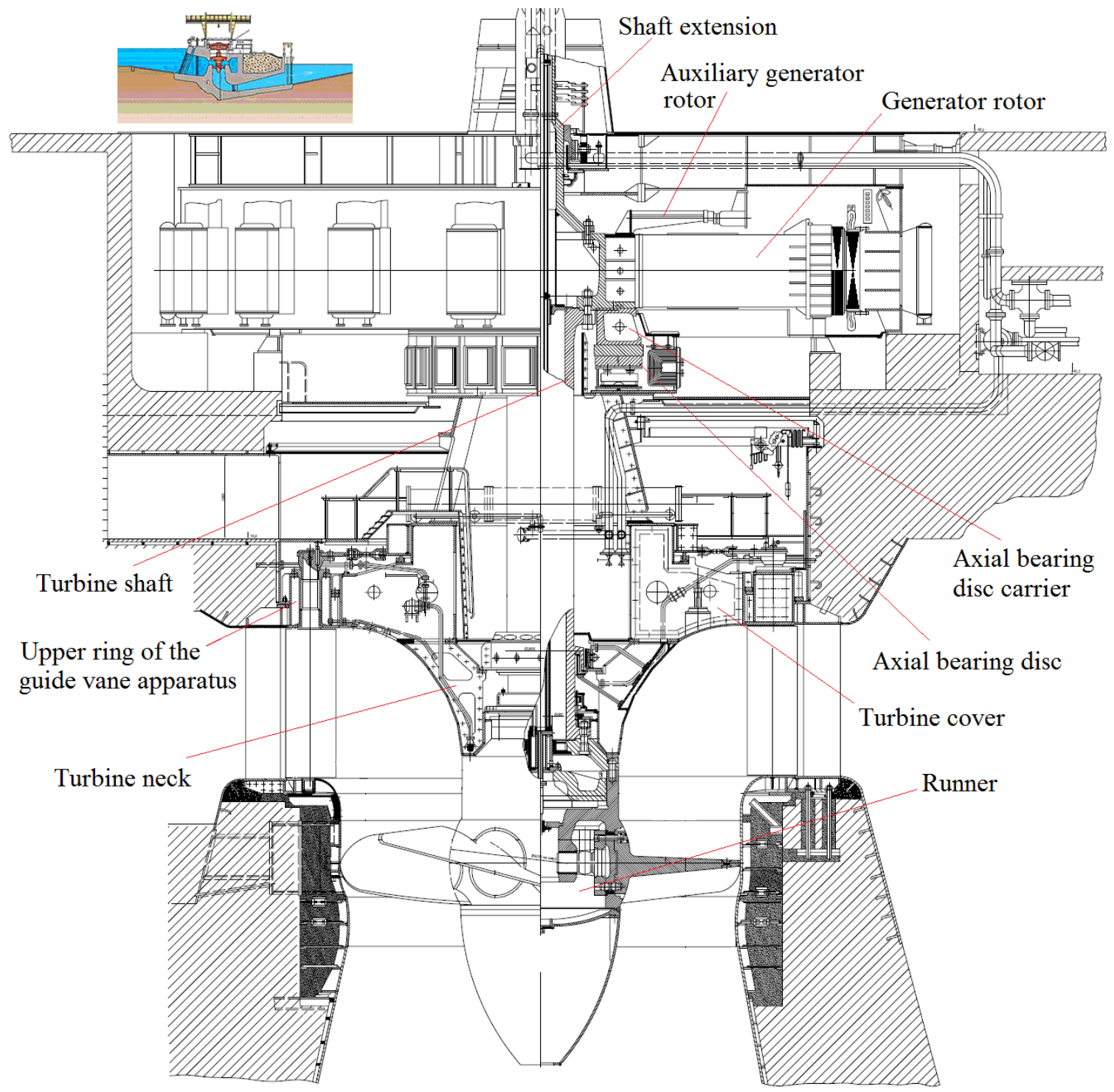

Fig.1. Appearance of the vertical Kaplan turbine, nominal power $200 \mathrm{MW}$

Hydro units should last for at least 40 years and/or 25000 hours before refurbishment. Average lifetime until refurbishment should not be less than 30 years long. Actual lifetime depends on maintenance quality and type of operation. It can be assumed that daily operation of the hydro unit lasts approximately $8-15$ hours.

Tests carried out on many hydro units showed that the magnitude of oscillations increases with the increase of operation hours. This can be caused by gradual erosion and corrosion of the hydro unit and it's bearings, as well as by abrasive and cavitational degradation of the turbine runner and turbine components, which disturbs the balance of the runner.

Technical Regulations and Standards should take into account the magnitude of oscillations as the main cause of crack propagation. Magnitude of oscillations directly influences the crack propagation velocity.

\subsection{Damaging and Degradation of Material Due to Fatigue}

Fatigue is progressive and localized damage of material structure that occurs when material is subjected to variable loads. Maximum values of stress that lead to fatigue are often significantly lower than the value of tensile strength, and sometimes even lower than the value of yield strength.

Problems linked with cavitation erosion, transitional states and vibrations could arise even after a several years of seemingly normal operation. These problems are in connection with catastrophic cases of facility damaging due to the failure of equipment, exceeded number of revolutions and similar causes. 


\subsection{Damaging and Degradation of Material Due to Corrosion}

Degradation of metal as a result of chemical or electrochemical action of the aggressive environment is called corrosion. Influence of corrosion on structural steels under conditions of high humidity is a process of material degradation due to the occurrence of initial cracks and their propagation because of variable loading. Taking the above mentioned into account, it can be said that maintenance and inspection are of highest importance. The overall number of initial cracks and high amplitudes could lead to failure in a short period of time, at a large number of loading cycles. Quantity of destroyed metal is in close connection with properties of the operating environment, pressure and temperature. Corrosion product is material with completely different physical and mechanical properties in comparison with initial properties of material.

\subsubsection{Damaging and Degradation of Material Due to Fatigue in Corrosive Environment}

Action of corrosion fatigue on structural steels of various quality is a process of material degradation due to the occurrence of initial cracks and their propagation, as well as due to cyclic loading. In some cases, one of these components can be more significant than the other (fatigue or corrosion), but generally speaking both components are significant. Maintenance and inspection are of highest importance because of that. At a large number of loading cycles, their overall number and high amplitudes can lead to failure in a brief period of time.

\subsection{Damaging and Degradation of Material Due to Erosion}

Existence of solid particles within the working fluid, which cannot be avoided, is the basic cause of destruction of metal surfaces. These particles originate due to metal corrosion, or come into work environment because of wearing of other parts. Rate of metal erosion, at room temperature, depends on numerous influential factors: particle dimensions, speed of particles, particle concentration, physical and mechanical properties of particles that wear the material, the material that's being worn, etc. In order to reduce the consequences of abrasive action of fluid, certain technical measures are being used: application of wear-resistant materials in order to extend service life, application of wear-resistant coatings and control of fluid flow by avoiding sharp transitions, bends, contractions, broadenings.

\subsection{Damaging and Degradation of Material Due to Cavitation}

Cavitation refers to the occurrence of cavities, or in other words to hollow areas at metal surface. Cavitation is one of liquid erosion mechanisms, which comprises creation and implosion of bubbles within the fluid. Process of elimination of material from the surface is called cavitation erosion, while resulting damage is called cavitation damage. Degraded surfaces of parts of hydraulic equipment subjected to cavitation can become rather large during short periods of time, while cavities can be up to $100 \mathrm{~mm}$ deep.

Cavitation damage at turbine and hydromechanical equipment originates as a result of degradation of surfaces at which the implosion of cavitation bubbles created when the critical speed is exceeded occurs, which mostly depends on the overall pressure. Process of degradation is in direct connection with conditions of exploitation. Cavitation damage is especially detectable at locations where the direction of streamlines and speed of liquid abruptly change.

Cavitation has not been investigated enough yet, but it is known that one of the causes for its origination are high speeds at which drops of liquid hit the surface of metal. Conditions necessary for the occurrence of critical speeds are being opstructed by structural solutions. Vibrations and noises mostly reveal the occurrence of cavitation damage at parts of turbine and hydromechanical equipment. At locations where cavitation occurs local pressures can reach $20 \mathrm{Mpa}$, which leads to mechanical degradation of material (creation of cavities and wearing). Cavitation causes the reduction of the positive effect of turbine operation, due to the fact that a substantial part of energy turns into heat.

\section{PRESSURE EQUIPMENT DIRECTIVES}

Pressure equipment or assemblies have to be designed in accordance with good engineering practice, in order to ensure safe operation. This equipment and/or assemblies have to be provided with adequate instruction manuals and have to carry manufacturers' identification marks. This equipment and/or assemblies must not be marked with compliance marks.

Before the pressure equipment hits the market, it has to be subjected to the prescribed compliance assessment procedure. Manufacturers can make a choice between the procedure based on manufacturing process / product inspection and procedure based on quality assurance system.

\subsection{Basic Requirements for Pressure Equipment Safety}

Pressure equipment has to be designed, constructed, tested and, if necessary, equipped and installed in such a way to ensure its safety when put into operation in compliance with manufacturers' manual or reasonably predictable conditions. In order to reach the most acceptable solutions, manufacturer has to obey certain principles in the following order:

- eliminate or reduce the possibility of hazardous occurrences as much as possible;

- apply certain safety measures regarding the hazardous occurrences which can't be eliminated;

- inform the user of existing hazards, as well as to take special measures in order to lower the risk during the installment and/or operation if necessary.

If there's a possibility of predicting the misuse which is the potential cause of danger, pressure equipment has to be designed in order to neutralize the possibility of misuse. 


\subsection{Basic Design Requirements}

Pressure equipment has to be designed for adequate loads according to its use and other predictable conditions. The following factors have to be taken into consideration:

- internal or external pressure,

- environmental temperature and operating temperature,

- static pressure and contents mass under operating and testing conditions,

- loads due to traffic, wind, earthquake,

- forces and moments induced by girders, supports, pipelines etc.

- corrosion, erosion, fatigue etc.,

- decomposition of unstable fluids.

Various loads can appear simultaneously and should be considered taking into account the possibility of their simultaneous appearance. Certain safety coefficients, as well as comprehensive methods, have to be used in order to determine adequate safety limits in respect to all known errors.

Allowable strains for pressure equipment have to be limited taking into account predictable errors under operating conditions. Safety coefficients have to be introduced in order to eliminate strength reduction induced during the production, as well as by operating conditions, strains and calculation models. Properties of the material, as well as its behaviour, also cause the reduction of strength.

The above mentioned requirements can be fulfilled through the application of one of the following methods, or through combination with other methods:

- design through the use of empirical formulas,

- design through the use of analitical procedures,

- design through the use of fracture mechanics.

\subsection{Basic Production Requirements}

Manufacturer has to provide proper implementation of all procedures that need to be fulfilled during the design process, as well as through the use of proper techniques and valid procedures, especially concerning the aspects presented in this paper.

Inseparable assembly of parts subjected to pressure and parts directly attached to them, as well as nondestructive testing, have to be performed by qualified personnel, in compliance with adequate work procedures.

\subsection{Basic Material Requirements}

Materials for parts subjected to pressure have:

- to possess appropriate properties for operating conditions which could be predicted, as well as for testing conditions. They have to be sufficiently ductile, strong and resistant to fracture.

- to be chemically resistant to fluids,

- to be aging resistant,

- to be convenient for adequate process procedures,

- to be chosen in such a way as to ensure that there are no major undesirable effects during the interaction of different materials.
Manufacturer has to provide elements that refer to the compliance with required material specifications, in one of the following ways:

- by using the materials in compliance with harmonized standards;

- by using the materials comprised by the approval for pressure equipment materials,

- by adequate material approvals.

\subsection{Harmonized Standards}

Harmonized standards are standards that refer to the same subject, approved by different standardization bodies, which enable the mutual replaceability of products, processes and services, or the mutual acceptance of testing results, as well as sharing of the information in accordance with JUS/ISO/IEC standards, Directive 2:2001. The relation between Pressure Equipment Directive and the Standard is presented in figure 2 .

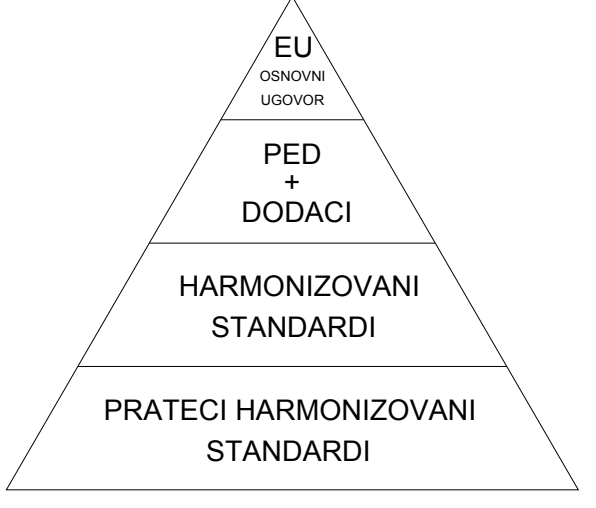

Fig. 2. Relation between the Directive and the Standard

Harmonized requirements contain technical requirements and define testing methods. Through the use of harmonized standards minimum safety requirements demanded by the Directive are fulfilled.

\section{MACHINERY DIRECTIVE}

Machinery Directive, which also refers to mechanical devices, defines obligations regarding the design, traffic and putting into operation of safe machines and the issuing procedure of CE markings. Directive 98/37/EEC and Appendix 98/79/EEC contain 4 Chapters and 9 Annexes.

They refer to:

- Manufacturers residing in EU Member States or

- Authorized representatives of a manufacturer with an office in one of EU Member States, or some other machine importer from the EU.

Those who complete the machines through the use of parts produced by different manufacturers are also obligated to follow the Machinery Directive, as well as those who produce machines for their personal use. Obligations also apply to manufacturers or machine parts produced to be installed into other machines, but standalone can`t function. Manufacturer or importer of such machines or devices has to issue the certificate which confirms that such products can be put into operation after the technical safety inspection of the whole machine into 
which they are supposed to be placed. Before putting the machine into operation manufacturer (or his authorized representative in the EU) has to provide technical documentation necessary for conformity assessment of products according to the requirements of the Directive and the Manual which is to be submitted along with the machine as an integral part of the product.

Conformity determination procedure, required by the Machinery Directive, is presented in figure 3. Directive 98/37/EEC and Appendix 98/79/EEC contain 4 Chapters and 9 Annexes.

Chapter I defines the objective, application area, goods traffic and free flow of goods. In this chapter it is stated that this Directive is applicable to machines, and in Appendix I appropriate requirements regarding safety and health are stated. Application area of the Machinery Directive also refers to safety parts which are being released into traffic. Exceptions to this rule are also stated for cases when the risk that refers to machines or safety components is covered by another Directive.
Chapter II defines conformity assessment procedures. Conformity assessment procedures, presented in this Chapter, refer to Appendix V - Declaration of Conformity and Appendix 6 - Type-examination.

Chapter III defines CE marking. CE conformity marking has to be in compliance with regulations stated in Appendix III. Safety components don't have CE markings. All machines and safety components have to be in compliance with EC Declaration of Conformity.

In Chapter IV regulations that refer to activities regarding the application of this Directive are defined.

Contents of the Declarations of Conformity, CE marking, types of machines and safety components, minimum criteria to be met by EU members for Certification Body registration and transitory Regulations are defined in Appendixes.

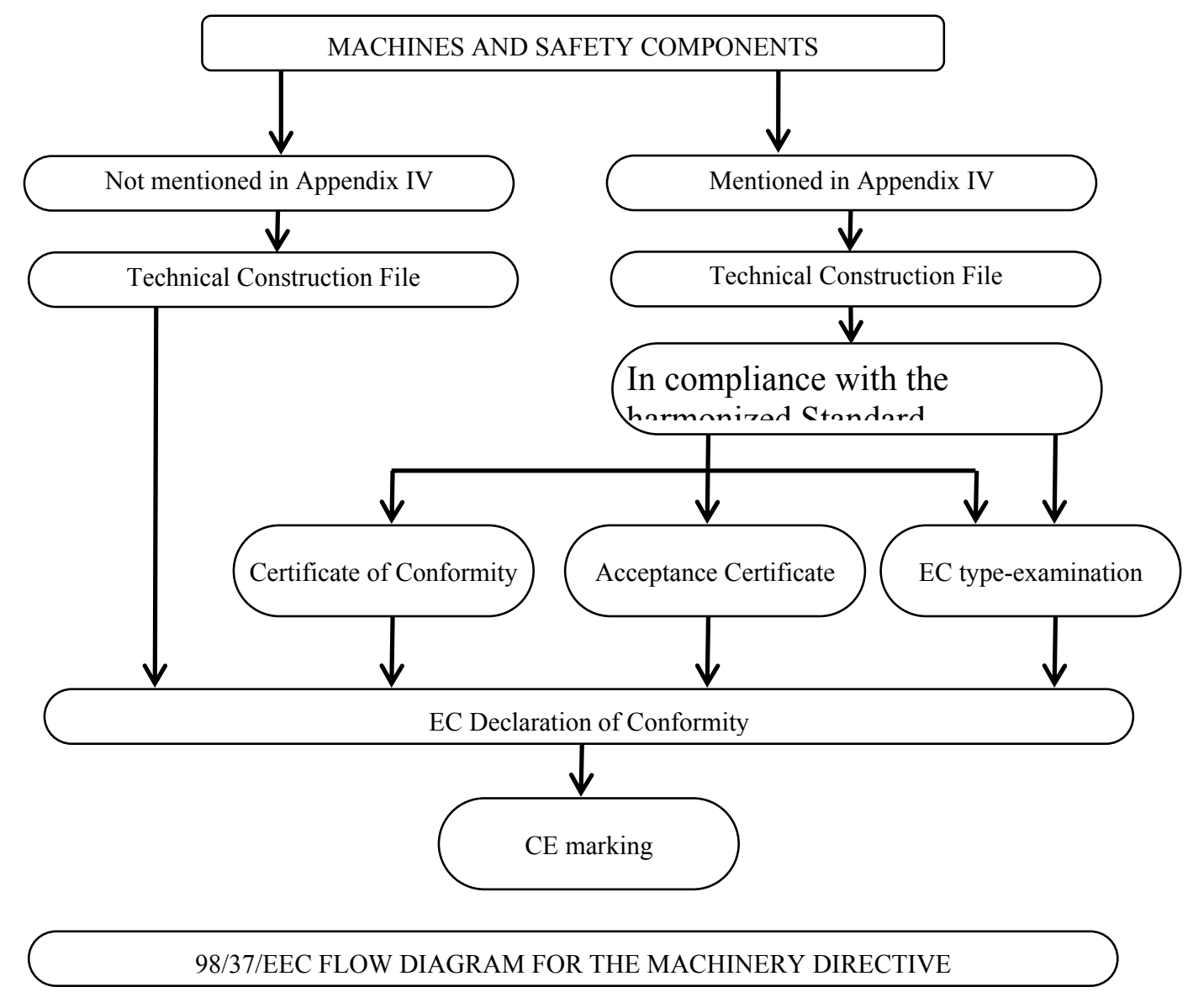

Fig.3. Chart that refers to conformity determination for machines and safety components

\subsection{Basic Requirements for the Safety of Machines}

Obligations derived from basic requirements for safety of machines and mechanical devices are also applicable to mechanical assemblies if certain danger exists. Basic requirements are obligatory, and they refer to:

- general (definitions, safety integration principles, materials and products, lighting, handling of machines),

- controlling and command devices,
- protective measures against mechanical hazards,

- requirements for protective devices,

- protective measures against other hazards,

- maintenance,

- general suggestions,

- food processing machines,

- hand-held and hand-guided machines, 
- machines for wood and similar materials processing,

- work locations,

- handling devices,

- protective measures against mechanical hazards,

- protective measures against other hazards,

- special requirements for non-mechanical lifting devices,

- markings,

- manual,

- low visibility hazards,

- freedom of movement,

- lighting,

- adjustment parts,

- movement limitation,

- fire danger,

- dust, gas hazard etc.

\subsection{Harmonized Standards}

Standardization program CEN/CENELEC, with respect to requirements of the 98/37/EEC Directive, is divided into several groups (categories) in order to avoid repetition and to enable the logical reasoning which would lead to quicker shaping of the Standard and connection to other Standards.
According to the adopted program of Standard hierarchy, they are divided into Standards of A, B and C type in the following fashion:

- Type A Standards (Basic Health and Safety Standards) provide the basic concept, design principles and general aspects and can be applied to all machines and mechanical devices, but refer to one aspect of health or safety which can be used for a wide range of machines.

- Type B1 Standards refer to singular safety aspects (e.g. surface temperature, noise, vibrations, safety distances);

- Type B2 Standards refer to safety devices (e.g. twohand controls, blockage devices, pressure-sensitive devices);

- Type C Standards refer to specific types of machines and provide detailed safety requirements for singular machines or groups of machines (e.g. semi-automatic machines, agricultural machines, food industry machines etc.)

It's important to point out that the basic objective of this Directive and similar Standards is to provide safety and health care. They refer not only to the concept, design, construction and production of machines, but to their use and utilization. The ultimate objective is to make sure that it's allowable to work only with machines which during exploitation, maintenance and repair do not threaten the operators.

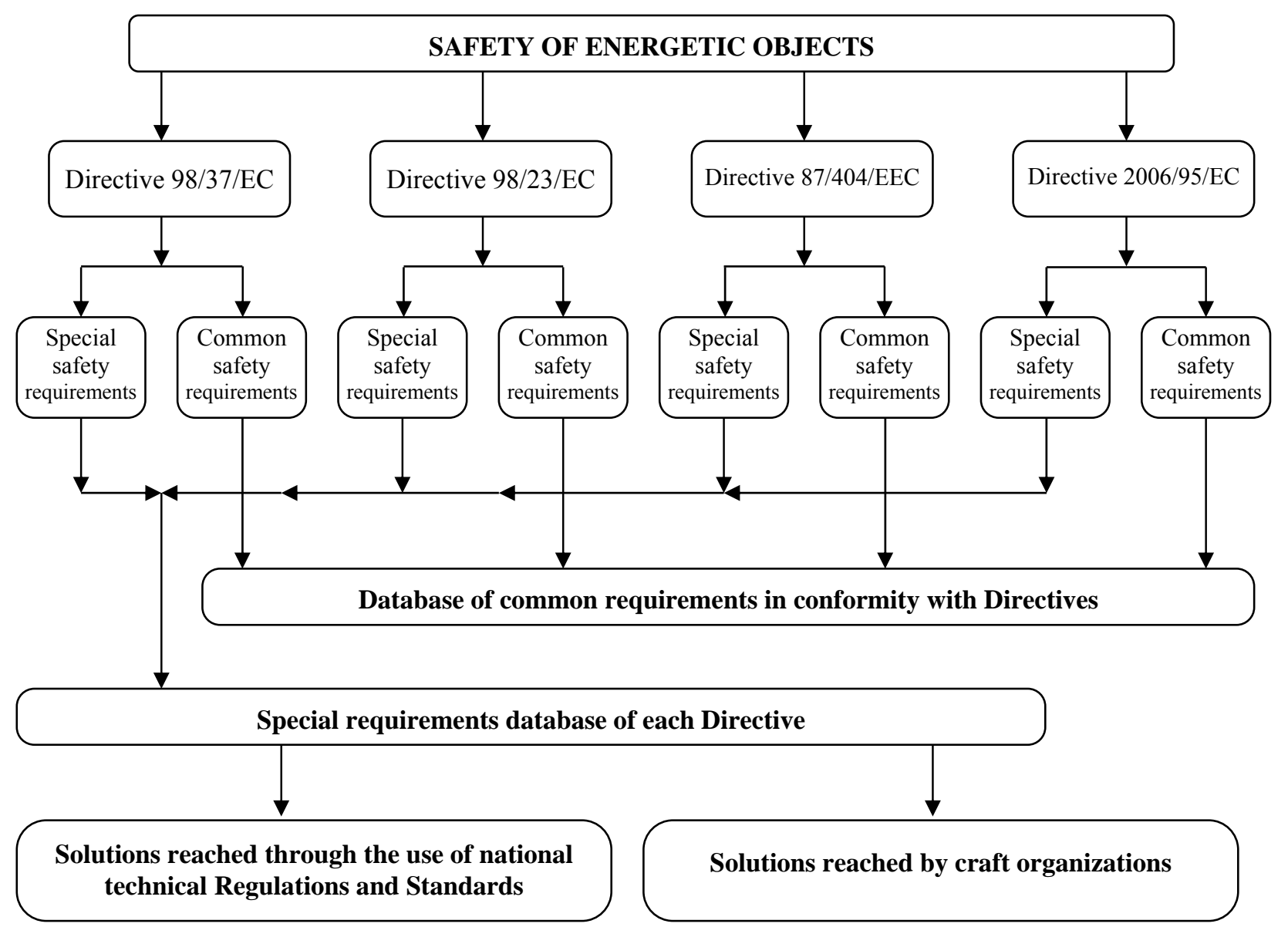

Fig.4. Complexity of EU Directives implementation in energetics chart 


\section{INSTEAD OF A CONCLUSION}

A large number of EU Directives and Technical Regulations define the safety of energetic objects, and that's why the energetic equipment manufacturer has to make sure that all equipment parts are in accordance with the requirements of the appropriate directive. Complexity of EU Directives implementation in energetics is obvious. The problem and the solution proposition are presented in figure 4.

\section{ACKNOWLEDGEMENT}

The authors acknowledge the support from the Serbian Ministry of Education, Science and Technological Development for Project TR 35002.

\section{REFERENCES}

[1] Manufacturer's Documentation for the vertical Kaplan turbine, LMZ, Sankt Petersburg

[2] The Pressure Equipment Directive 97/23/EC

[3] The Machinery Directive 98/37/EEC

[4] Arsić M., Vistać B., Odanović Z., Đorđević A., Savić Z., "Repair Welding Methodology for the Turbine Shaft at the Hydroelectric Power Plant Djerdap 2 Without Disassembling The Hydroelectric Generating Set," IMK 14 - Research\&Development, Vol. 18, No. 3, 2012

[5] Bižić M., Petrović D., "Identification of the Effects of Circular Hole on the Stress State of Homogeneous Isotropic Uniaxial Tensioned Plate" IMK 14 Research\&Development, Vol. 17, No. 3, 2011

[6] Alămoreanu M., "Evaluation of the available resources of the self-supporting structures of hoisting machines during exploitation," IMK 14 Research\&Development, Vol. 19, No. 2, 2013 


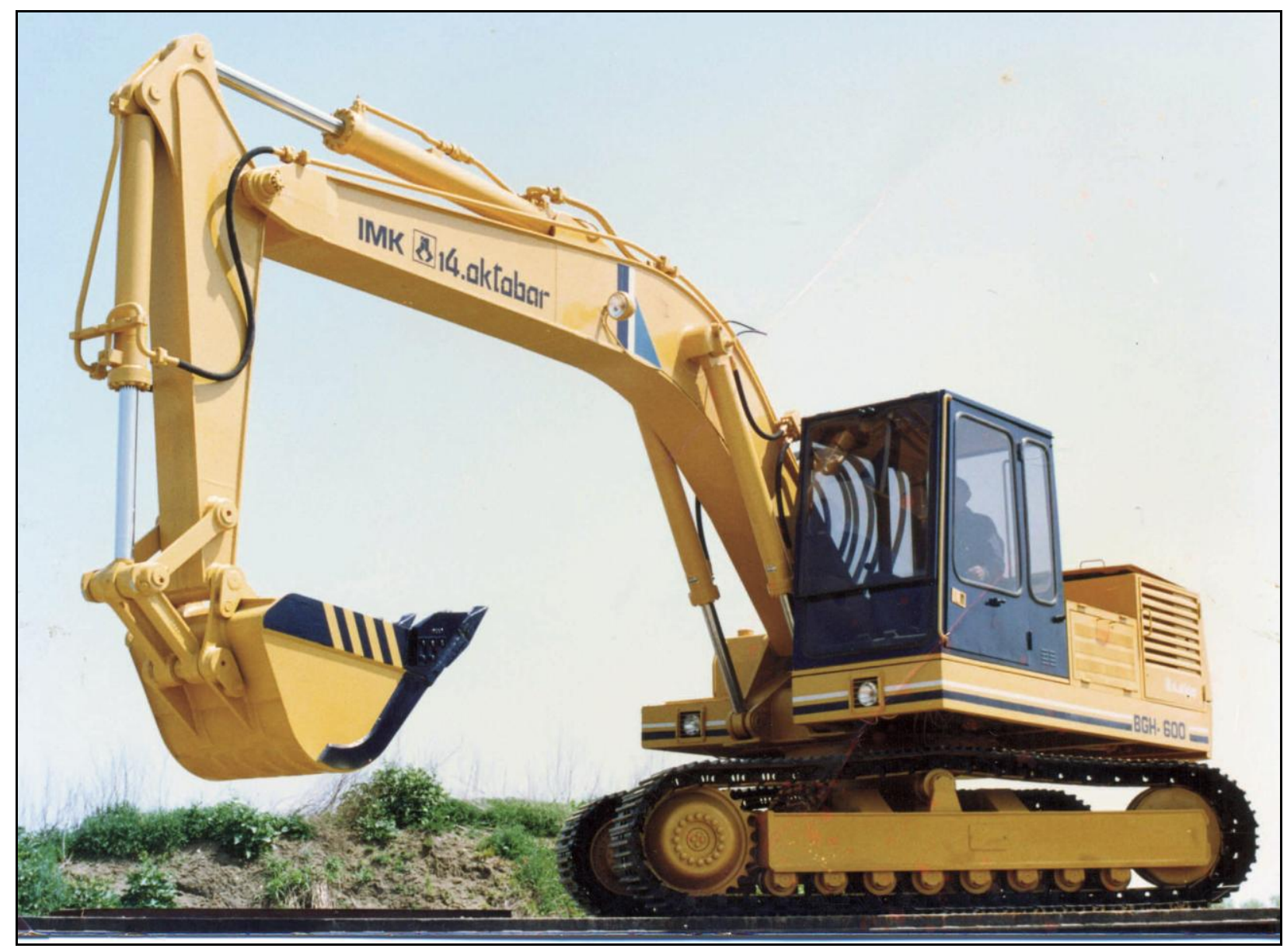

